Supporting Information for

\title{
Highly Anisotropic Corncob as Efficient Solar Steam Generation Device with Heat Localization and Rapid Water Transportation
}

Tingjie Chen, ${ }^{\dagger},+, \perp, *$ Hao Xie, ${ }^{\dagger}, \perp$ Xin Qiao, $\dagger$ Shuqiang Hao, ${ }^{\dagger}$ Zhenzeng Wu,,$^{\S}$ Duo Sun,${ }^{\dagger}$ Zhiyong Liu, ${ }^{\ddagger}$ Fang Cao,${ }^{\dagger}$ Binghui $\mathrm{Wu},{ }^{\dagger}$ and Xiaoliang Fang, ${ }^{\dagger}, *$

${ }^{\dagger}$ Collaborative Innovation Center of Chemistry for Energy Materials, Department of Chemistry, College of Chemistry and Chemical Engineering, Pen-Tung Sah Institute of Micro-nano Science and Technology, Graphene Industry and Engineering Research Institute, Xiamen University, Xiamen, Fujian 361005, P. R. China.

$\$$ College of Materials Science and Engineering, Key Laboratory of Polymer Materials and Products of Universities in Fujian, Fujian University of Technology, Fuzhou, Fujian 350002, P.R. China.

$\S$ College of Ecology and Resource Engineering, Wuyi University, No. 16, Wuyi Avenue, Wuyishan City, Fujian 354300, P.R. China

$\perp$ Tingjie CHEN and Hao XIE contributed equally to this work

\section{* Corresponding Author:}

Tingjie Chen, E-mail: phdctj2014@163.com;

Xiaoliang Fang, E-mail: x.l.fang@xmu.edu.cn. 

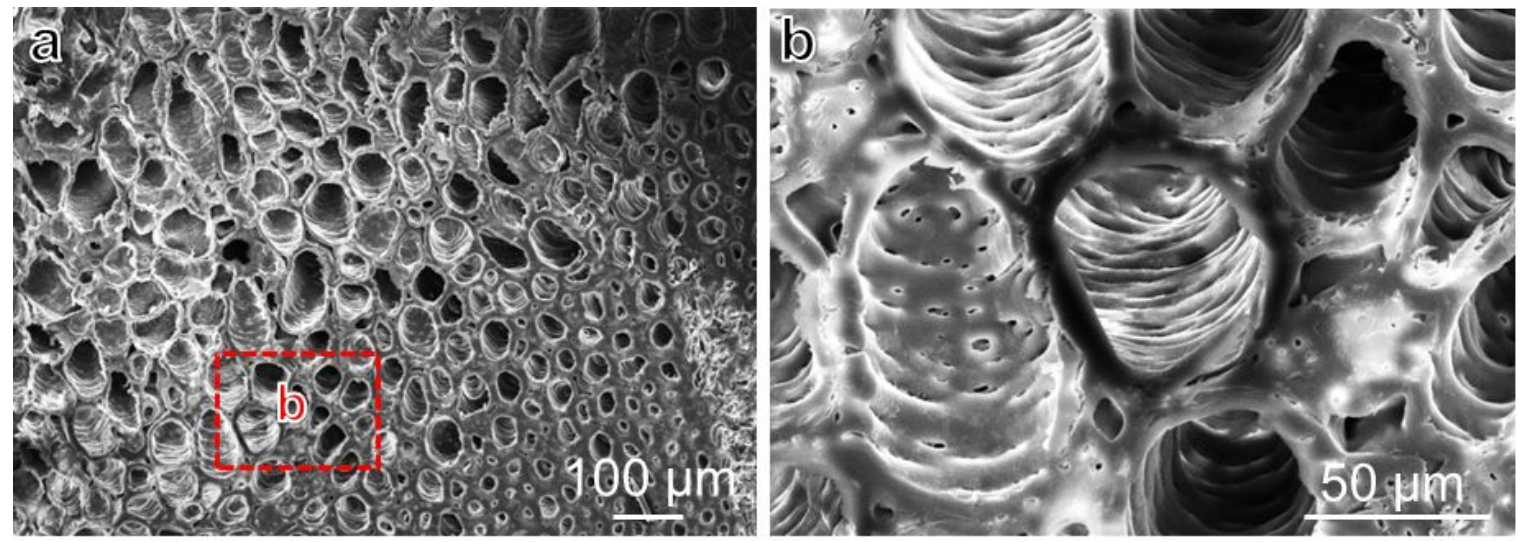

Figure S1. (a) Cross-section image of the outer part of natural corncob showing highly porous

microstructures and (b) Magnification image from the frame in (a).

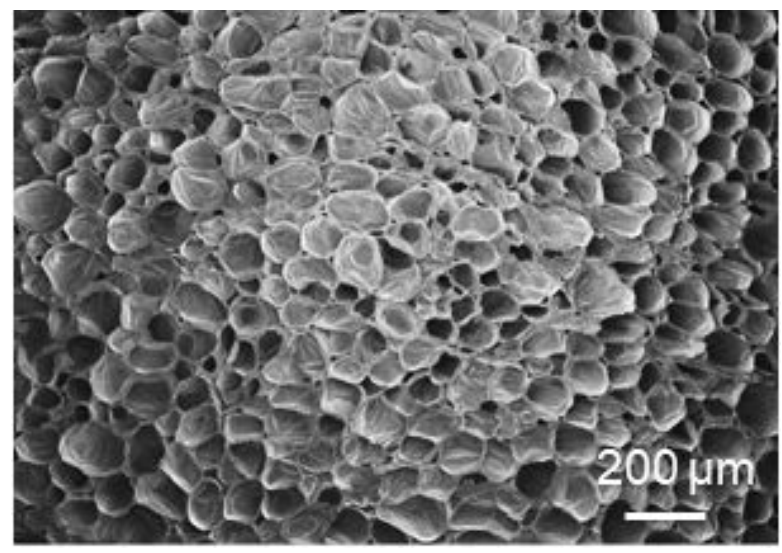

Figure S2. Cross-section image of the central part of natural corncob.
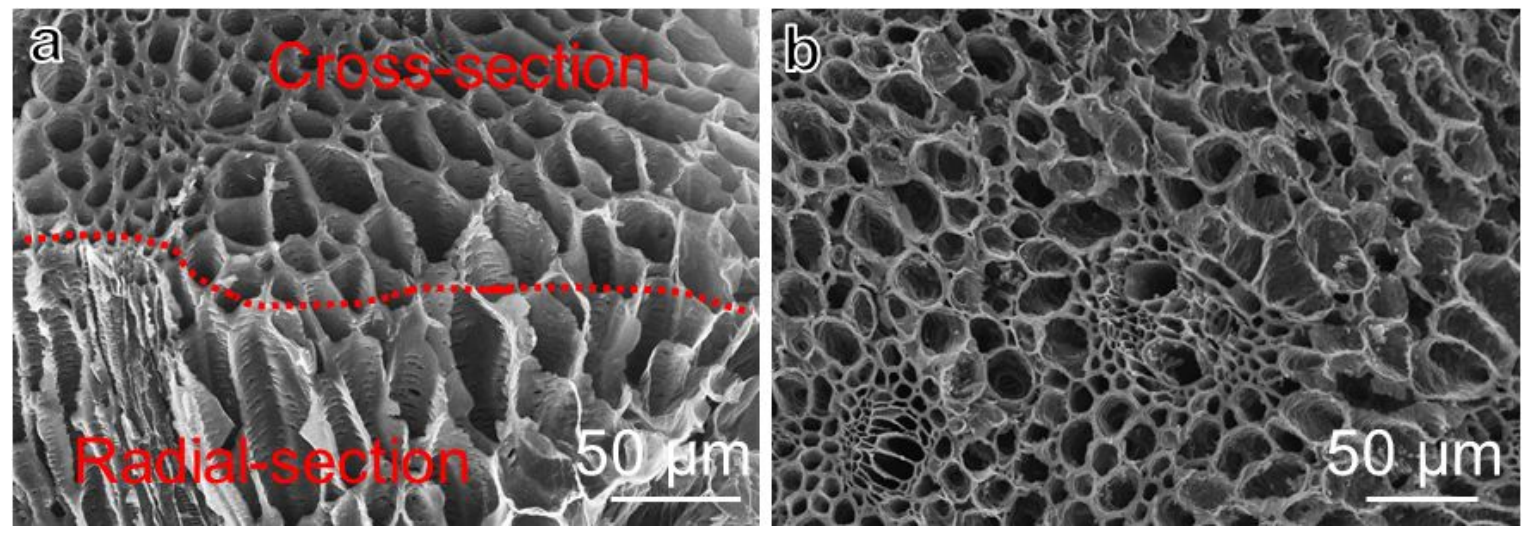

Figure S3. (a) Radial and cross-section and (b) Cross-section images of the outer part of carbonized corncob. 

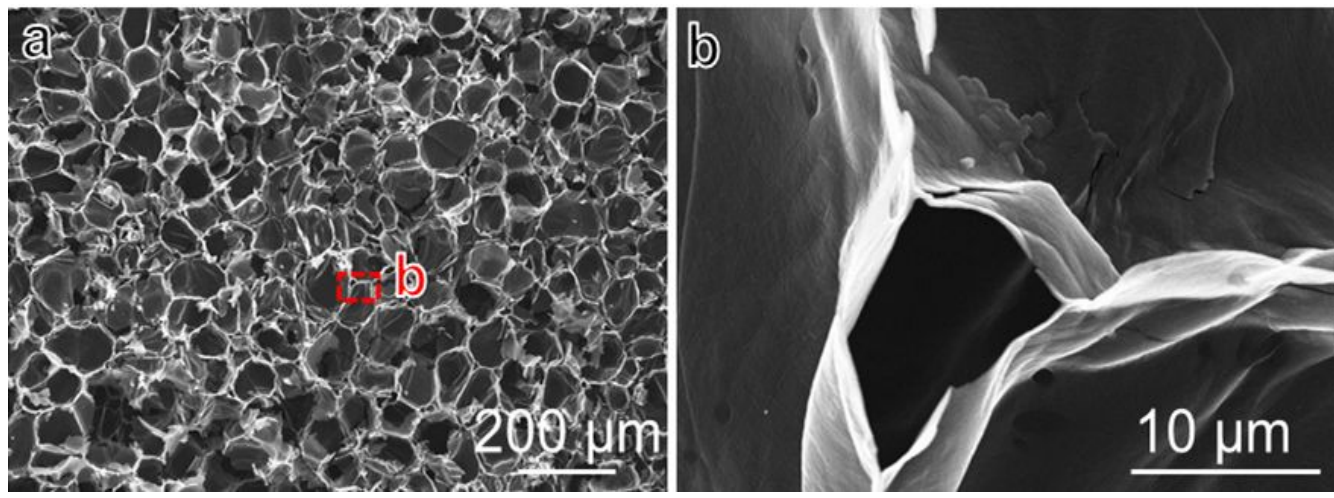

Figure S4. (a) Cross-section images of the central part of carbonized corncob and (b)

Magnification image from the frame in (a).
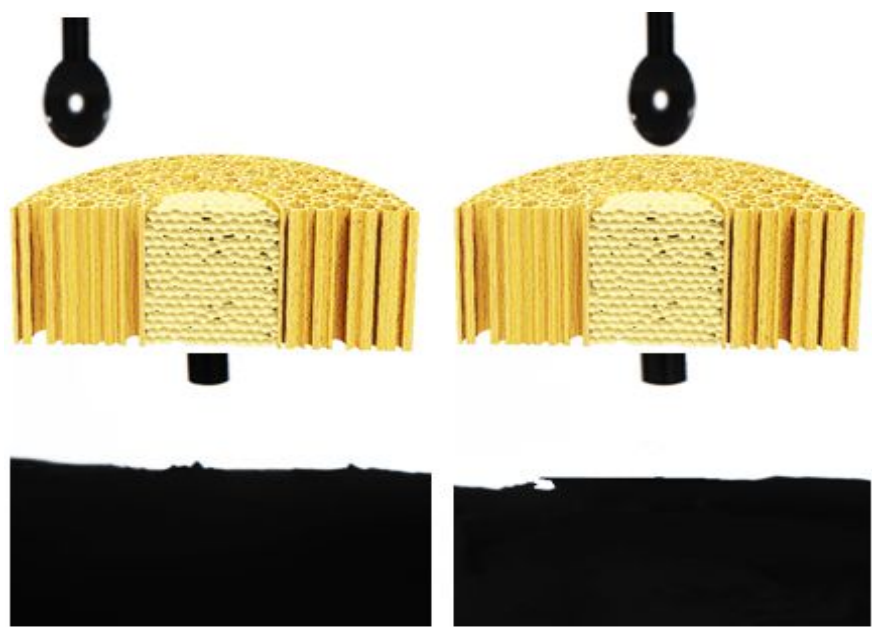

Figure S5. Contact angles of the outer and central parts of natural corncob.
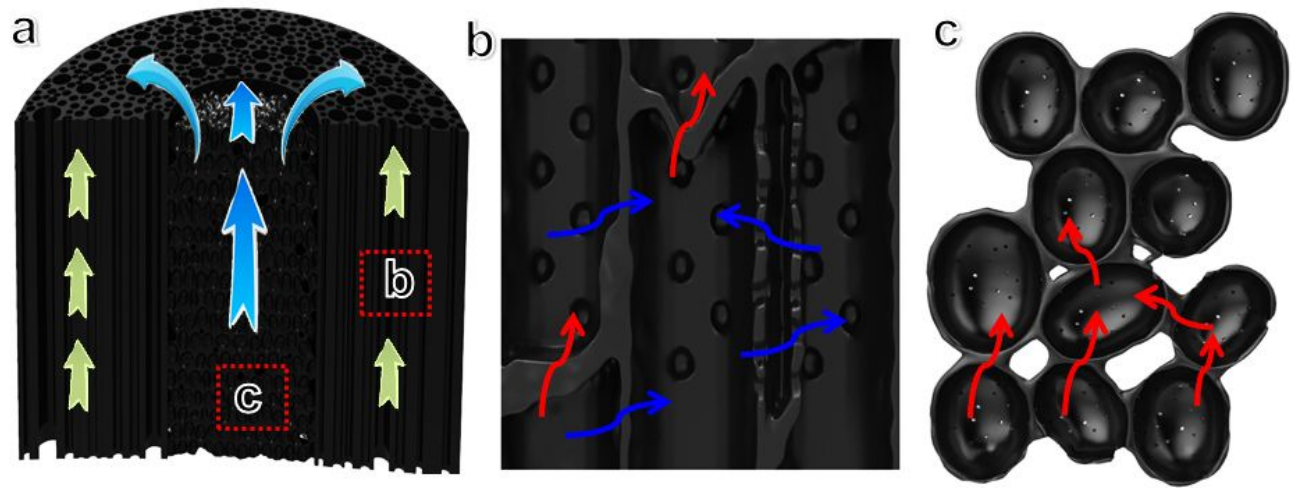

Figure S6. (a) Schematic to illustrate the water transportation capability in the outer and central parts of C-corncob; (b) and (c) Magnification images from the frame in (a). 


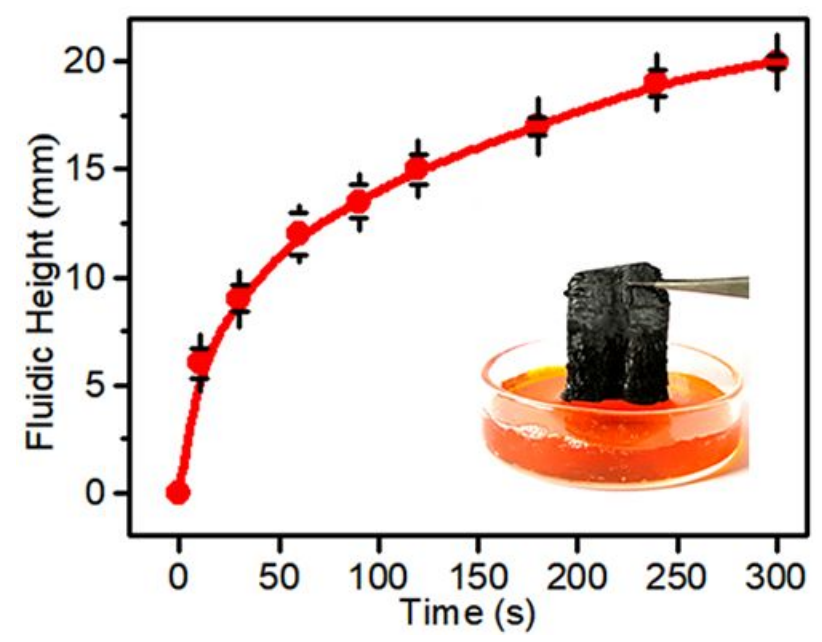

Figure S7. The transportation of the methyl-orange aqueous solution by C-corncob.

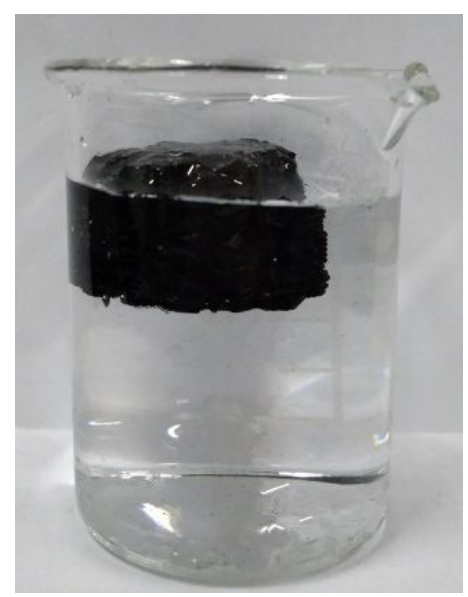

Figure S8. Images showing the C-corncob device floating in seawater.
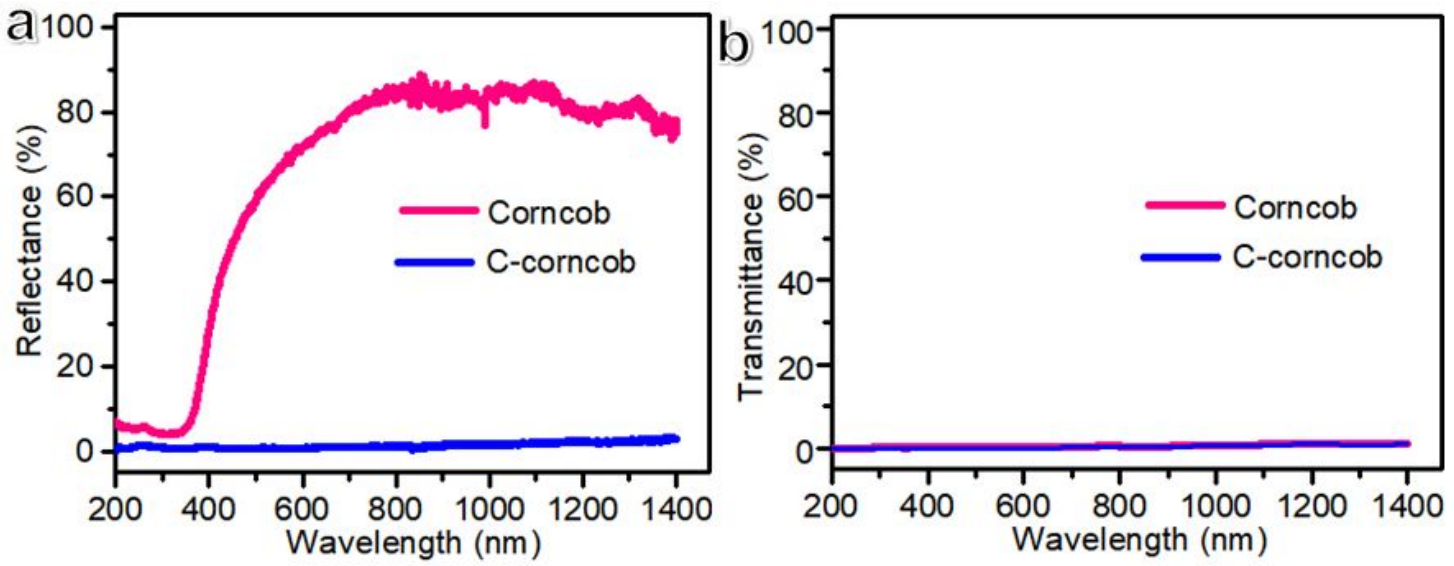

Figure S9. (a) The reflectance and (b) Transmittance spectra of corncob and C-corncob. 


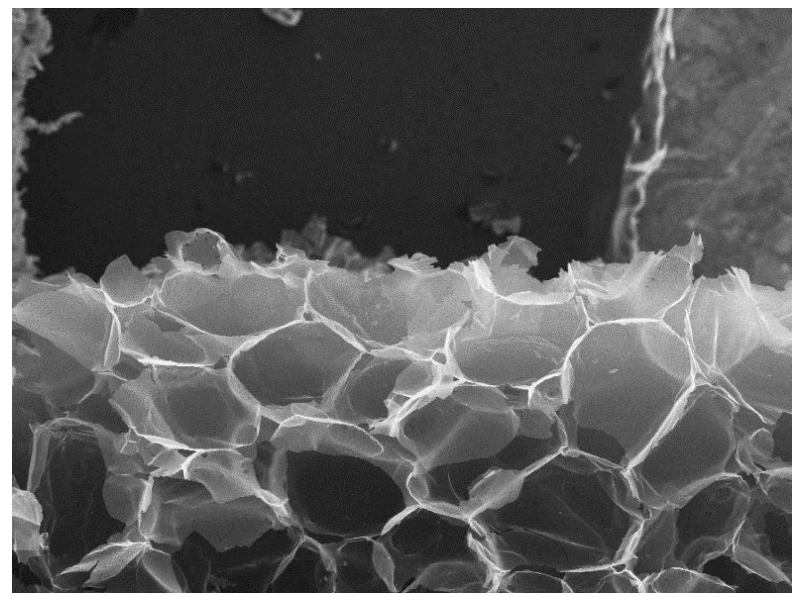

Figure S10. SEM image shows the C-corncob with rough surface.

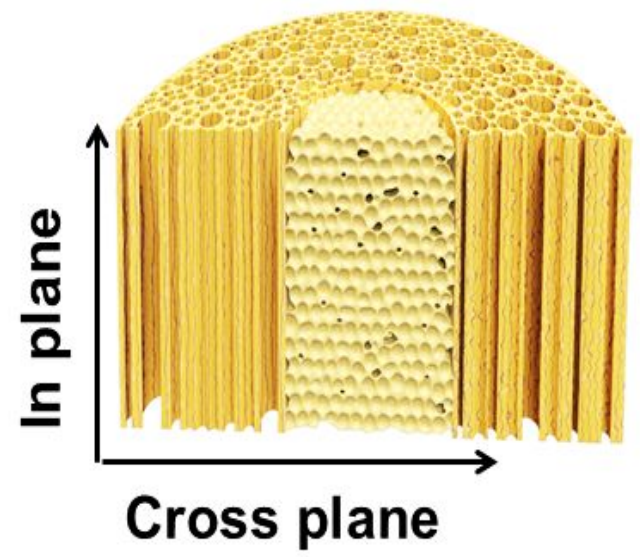

Figure S11. Schematic to illustrate the two planes of a radial section of corncob.

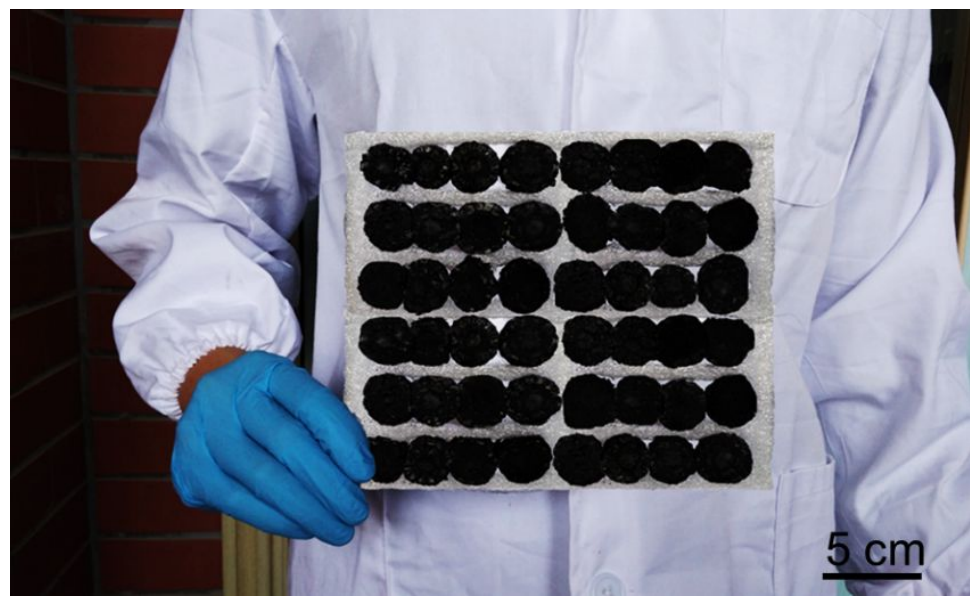

Figure S12. Photograph of a large piece of as-prepared C-corncob device. 


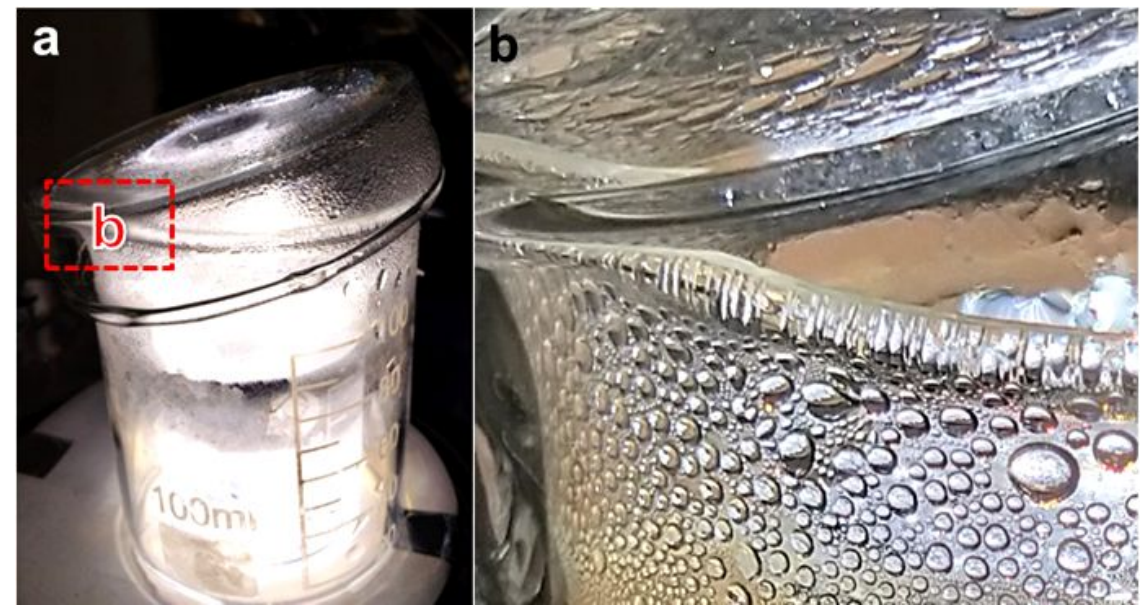

Figure S13. (a) The optical image of the condensed water on the inner side of the glassware and (b) Magnification image showing the condensed water from the frame in (a).
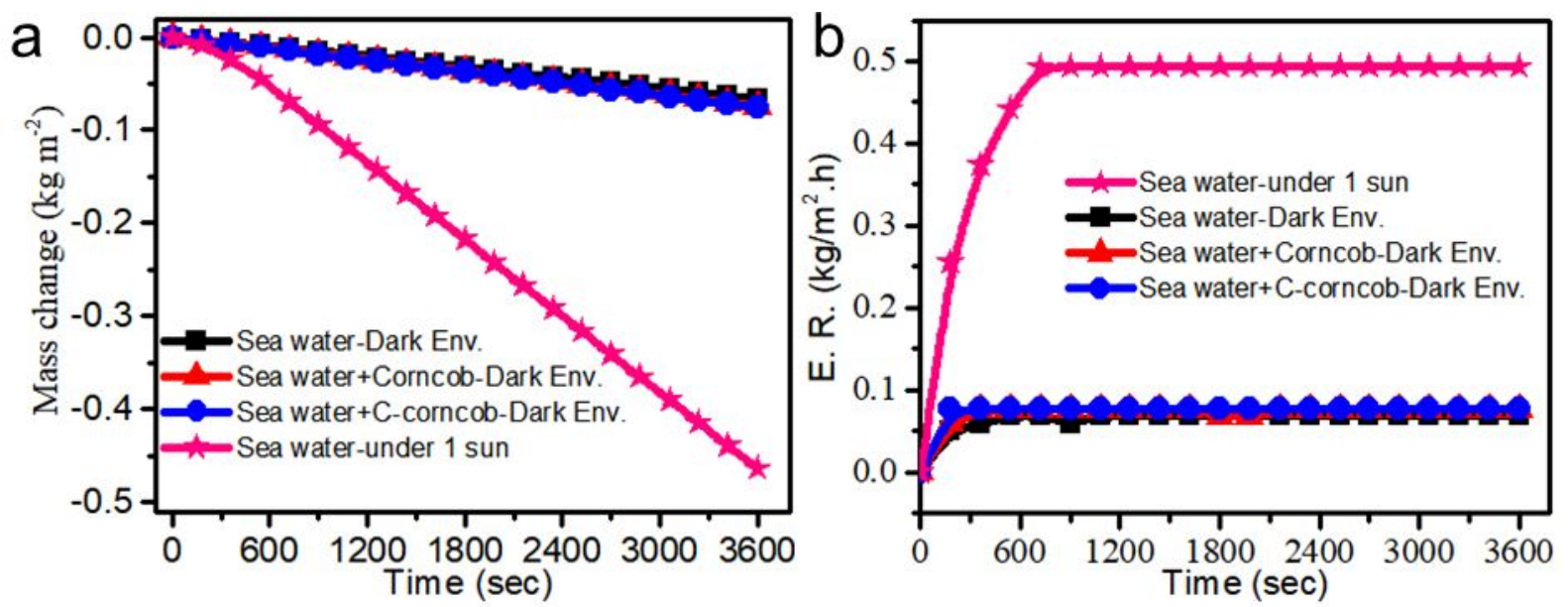

Figure S14. (a) Weight changes and (b) Evaporation rate evolutions of seawater under solar irradiation of $1 \mathrm{~kW} \mathrm{~m}^{-2}$ and three samples (i.e., seawater, corncob and C-corncob) under dark condition. The intrinsic evaporating abilities of seawater, corncob, and C-corncob under dark condition are about $0.068 \mathrm{~kg} \mathrm{~m}^{-2} \mathrm{~h}^{-1}, 0.076 \mathrm{~kg} \mathrm{~m}^{-2} \mathrm{~h}^{-1}$, and $0.076 \mathrm{~kg} \mathrm{~m}^{-2} \mathrm{~h}^{-1}$, respectively. 


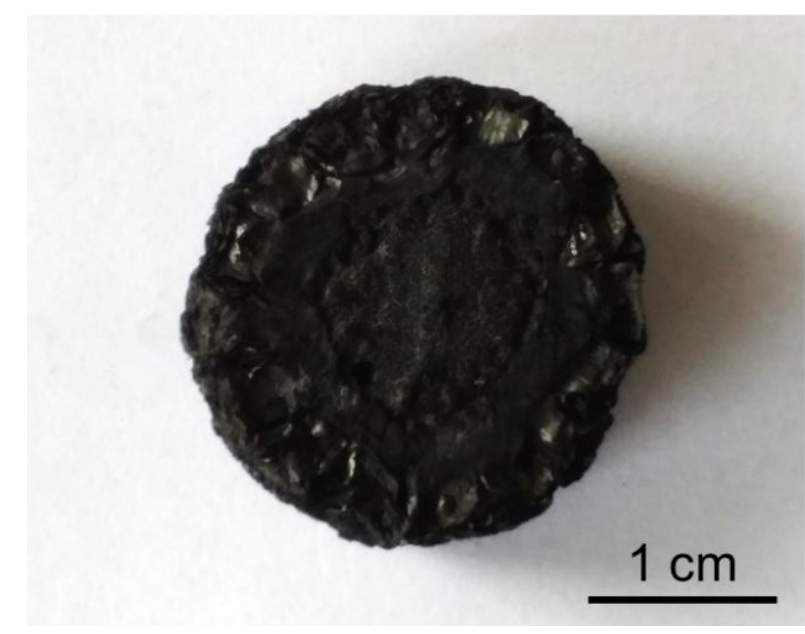

Figure S15. Image of C-corncob after one sun desalination for $360 \mathrm{~min}$.
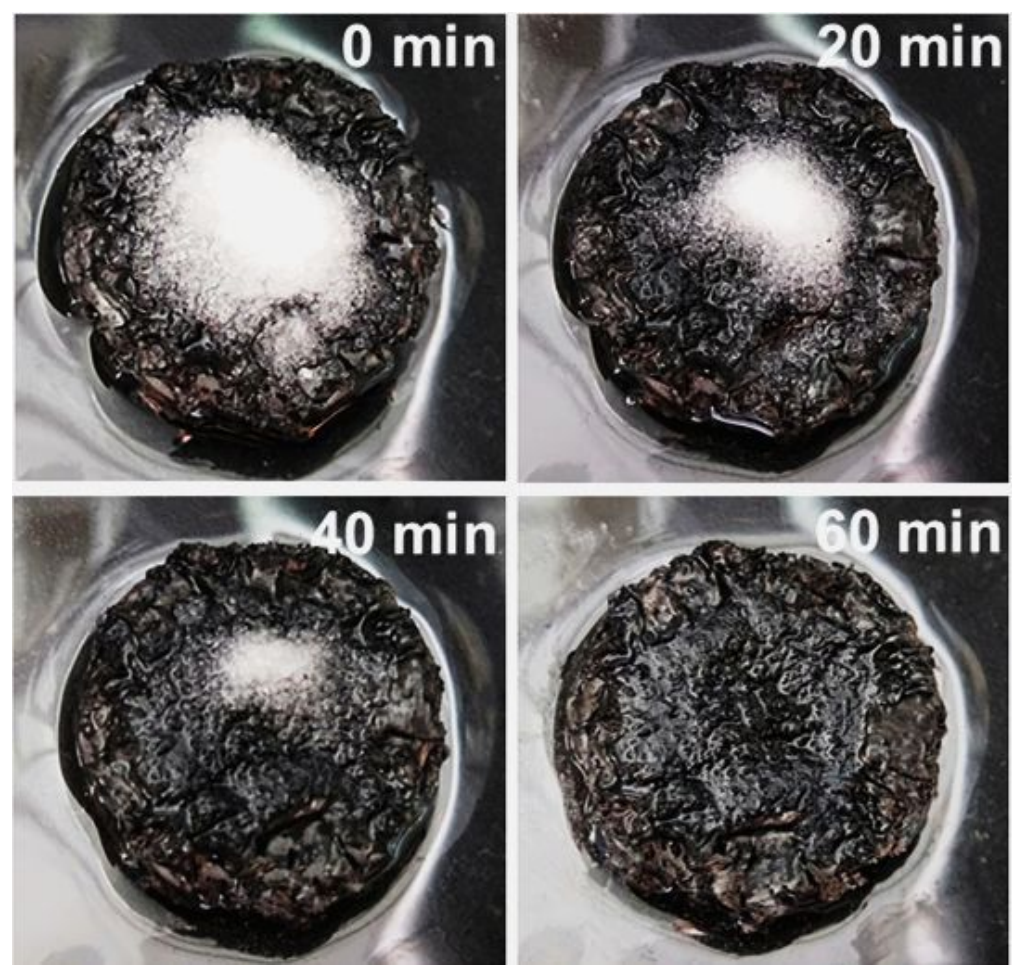

Figure S16. Images show the progress of solid salt $(0.5 \mathrm{~g} \mathrm{NaCl})$ infiltration back to the bulk brine solution $(3.5 \% \mathrm{NaCl}$ solution) under 1 sun illumination. This experiment indicates the excellent salt rejection ability of porous C-corncob. 
Table S1. Comparison of the efficiency of our work to ever-reported works under 1 sun illumination.

\begin{tabular}{|c|c|c|c|c|}
\hline Material & $\begin{array}{c}\text { ERs } \\
\left(\mathrm{kg} \mathrm{m}^{-2} \mathrm{~h}^{-1}\right)\end{array}$ & $\begin{array}{c}\text { Steam- } \\
\text { generation } \\
\text { efficiency }\end{array}$ & Scalability & Ref. \\
\hline Carbonized corncob & 1.36 (net) & $86.7 \%$ & Large & $\begin{array}{l}\text { This } \\
\text { work }\end{array}$ \\
\hline $\begin{array}{l}\text { Double-layer structure (DLS) consists of a } \\
\text { carbon foam layer supporting an exfoliated } \\
\text { graphite layer }\end{array}$ & - & $64 \%$ & Medium & 1 \\
\hline $\begin{array}{l}\text { Carbon nanotube (CNT)-modified flexible wood } \\
\text { membrane }\end{array}$ & 0.95 (net) & $65 \%$ & Medium & 2 \\
\hline $\begin{array}{l}\text { Surface-carbonized longitudinal wood (C-L- } \\
\text { Wood) }\end{array}$ & 1.08 (net) & $74 \%$ & Large & 3 \\
\hline Carbon nanotube (CNT)-modified filter paper & 1.1 & $75 \%$ & Medium & 4 \\
\hline Wasted rice straw & 1.2 & $75.8 \%$ & Medium & 5 \\
\hline $\begin{array}{l}\text { Bilayer aerogel structure employing cellulose } \\
\text { nanofibrils (CNFs) and carbon nanotube (CNT) } \\
\text { layer }\end{array}$ & 1.11 & $76.3 \%$ & Medium & 6 \\
\hline Carbonized mushrooms & 1.28 (net) & $78 \%$ & Medium & 7 \\
\hline PAN coated with Au nano particles & - & $79.3 \%$ & Small & 8 \\
\hline $\begin{array}{l}\text { Commercially available activated carbon fiber } \\
\text { felt }\end{array}$ & 1.02 (net) & $79.4 \%$ & Large & 9 \\
\hline $\begin{array}{l}\text { 2D water path design consists of graphene oxide } \\
\text { and cellulose wrapped polystyrene foam }\end{array}$ & 1.45 & $80 \%$ & Medium & 10 \\
\hline PEGylated $\mathrm{MoS}_{2}$-cotton cloth & 1.3 & $80.1 \%$ & Large & 11 \\
\hline $\begin{array}{l}\text { Bi-layered reduced graphene oxide film on } \\
\text { polystyrene foam }\end{array}$ & 1.31 & $83 \%$ & Medium & 12 \\
\hline Multifunctional $\mathrm{CuO}$ Nanowire Mesh & 1.42 & $84.4 \%$ & Small & 13 \\
\hline $\begin{array}{l}\text { Graphene oxide (GO)-based aerogel consists of } \\
\text { GO, sodium alginate (SA), and multiwalled } \\
\text { carbon nanotubes }\end{array}$ & 1.62 & $85 \%$ & Medium & 14 \\
\hline $\begin{array}{l}\text { 3D-printed evaporator consists of the } \mathrm{CNT} / \mathrm{GO} \\
\text { layer, GO/nanofibrillated cellulose (NFC) layer, } \\
\text { and GO/NFC wall }\end{array}$ & 1.25 (net) & $85.6 \%$ & Medium & 15 \\
\hline Nitrogen doped graphene aerogel & 1.34 (net) & $86.2 \%$ & Medium & 16 \\
\hline $\begin{array}{l}\text { 3D cross-linked honeycomb graphene foam } \\
\text { material }\end{array}$ & 1.30 (net) & $87 \%$ & Small & 17 \\
\hline $\begin{array}{l}\text { Surface-carbonized de-sugaring stems of } \\
\text { sugarcane }\end{array}$ & 1.31 (net) & $87.4 \%$ & Large & 18 \\
\hline $\begin{array}{l}\text { Foam-strengthened ultrablack carbon aerogels } \\
\text { (CAs) }\end{array}$ & 1.37 & $87.5 \%$ & Large & 19 \\
\hline Phenolic aldehyde foams & 1.27 (net) & $87.86 \%$ & Small & 20 \\
\hline $\begin{array}{l}\text { Reduced graphene oxide wrapped plant fiber } \\
\text { sponges }\end{array}$ & 1.34 (net) & $88.8 \%$ & Large & 21 \\
\hline $\begin{array}{l}\text { Oil lamp inspired design, cotton strand coated } \\
\text { lpolydopamine }\end{array}$ & 1.28 (net) & $88.8 \%$ & Medium & 22 \\
\hline Graphene/Rice-Straw Aerogels & 1.40 (net) & $88.9 \%$ & Large & 23 \\
\hline
\end{tabular}




\begin{tabular}{|c|c|c|c|c|}
\hline Wood treated by tannin acid (TA)- $-\mathrm{Fe}^{3+}$ & 1.43 (net) & $90 \%$ & Large & 24 \\
\hline $\mathrm{MoS}_{2}$-loaded carbon foam & 1.28 (net) & $90.4 \%$ & Large & 25 \\
\hline Wood treated by aluminophosphate & 1.42 (net) & $90.8 \%$ & Large & 26 \\
\hline $\begin{array}{l}\text { Graphdiyne (GDY)-based hierarchical } \\
\text { architecture }\end{array}$ & 1.45 (net) & $91 \%$ & Small & 27 \\
\hline Single-walled nanotube--MoS ${ }_{2}$ hybrid film & - & $91.5 \%$ & Small & 28 \\
\hline Calcination of melamine sponges (MS) in air & 1.98 & $92 \%$ & Large & 29 \\
\hline PAN and PAN/GO bilayer membrane & - & $92.6 \%$ & Medium & 30 \\
\hline Carbon nanomaterial coated on fabric & 1.42 (net) & $93 \%$ & Small & 31 \\
\hline CuxS-based solar water evaporator & 1.73 (net) & $94.5 \%$ & Small & 32 \\
\hline $\begin{array}{l}\text { Macroporous vertically aligned graphene pillar } \\
\text { array }\end{array}$ & 1.47 (net) & $95 \%$ & Small & 33 \\
\hline
\end{tabular}

\section{References}

(S1) Ghasemi, H.; Ni, G.; Marconnet, A.; Loomis, J.; Yerci, S.; Miljkovic, N.; Chen, G. Solar Steam Generation by Heat Localization. Nat. Commun. 2014, 5, 4449.

(S2) Chen, C.; Li, Y.; Song, J.; Yang, Z.; Kuang, Y.; Hitz, E.; Jia, C.; Gong, A.; Jiang, F.; Zhu, J.; Yang, B.; Xie, J.; Hu, L. Highly Flexible and Efficient Solar Steam Generation Device. Adv. Mater. 2017, 29, 1701756.

(S3) Liu, H.; Chen, C.; Chen, G.; Kuang, Y.; Zhao, X.; Song, J.; Jia, C.; Xu, X.; Hitz, E.; Xie, H.; Wang, S.; Jiang, F.; Li, T.; Li, Y.; Gong, A.; Yang, R.; Das, S.; Hu, L. High-Performance Solar Steam Device with Layered Channels: Artificial Tree with a Reversed Design. Adv. Energy Mater. 2018, 8, 1701616.

(S4) Yang, P.; Liu, K.; Chen, Q.; Li, J.; Duan, J.; Xue, G.; Xu, Z.; Xie, W.; Zhou, J. Solar-Driven Simultaneous Steam Production and Electricity Generation from Salinity. Energy Environ. Sci. 2017, 10, 1923-1927.

(S5) Fang, Q.; Li, T.; Chen, Z.; Lin, H.; Wang, P.; Liu, F. Full Biomass-Derived Solar Stills for Robust and Stable Evaporation to Collect Clean Water from Various Water-Bearing Media. ACS Appl. Mater. Interfaces 2019, 11, 10672-10679.

(S6) Jiang, F.; Liu, H.; Li, Y.; Kuang, Y.; Xu, X.; Chen, C.; Huang, H.; Jia, C.; Zhao, X.; Hitz, E.; Zhou, Y.; Yang, R.; Cui, L.; Hu, L. Lightweight, Mesoporous, and Highly Absorptive All-Nanofiber Aerogel for Efficient Solar Steam Generation. ACS Appl. Mater. Interfaces 2018, 10, 1104-1112.

(S7) Xu, N.; Hu, X.; Xu, W.; Li, X.; Zhou, L.; Zhu, S.; Zhu, J. Mushrooms as Efficient Solar Steam-Generation Devices. Adv. Mater. 2017, 29, 1606762.

(S8) Huang, Z.; Li, S.; Cui, X.; Wan, Y.; Xiao, Y.; Tian, S.; Wang, H.; Li, X.; Zhao, Q.; Lee,C. A Broadband Aggregation-Independent Plasmonic Absorber for Highly Efficient Solar Steam Generation. J. Mater. Chem. A 2020, 8, 10742-10746.

(S9) Li, H.; He, Y.; Hu, Y.; Wang, X. Commercially Available Activated Carbon Fiber Felt Enables Efficient Solar Steam Generation. ACS Appl. Mater. Interfaces 2018, 10, 9362-9368.

(S10) Li, X.; Xu, W.; Tang, M.; Zhou, L.; Zhu, B.; Zhu, S.; Zhu, J. Graphene Oxide-Based Efficient and Scalable Solar Desalination under One Sun with a Confined 2D Water Path. Proc. Natl. Acad. Sci. 2016, 113, 13953-13958. (S11) Guo, Z.; Wang, G.; Ming, X.; Mei, T.; Wang, J.; Li, J.; Qian, J.; Wang, X. PEGylated Self-Growth $\mathrm{MoS}_{2}$ on a Cotton Cloth Substrate for High-Efficiency Solar Energy Utilization. ACS Appl. Mater. Interfaces 2018, 10, 24583-24589.

(S12) Shi, L.; Wang, Y.; Zhang, L.; Wang, P. Rational Design of a Bi-Layered Reduced Graphene Oxide Film on Polystyrene Foam for Solar-Driven Interfacial Water Evaporation. J. Mater. Chem. A 2017, 5, 16212-16219.

(S13) Xu, Y.; Ma, J.; Han, Y.; Zhang, J.; Cui, F.; Zhao, Y.; Li,; Wang, W. Multifunctional CuO Nanowire Mesh for Highly Efficient Solar Evaporation and Water Purification. ACS Sustainable Chem. Eng. 2019, 7, 5476-5485. 
(S14) Hu, X.; Xu, W.; Zhou, L.; Tan, Y.; Wang, Y.; Zhu, S.; Zhu, J. Tailoring Graphene Oxide-Based Aerogels for Efficient Solar Steam Generation under One Sun. Adv. Mater. 2017, 29, 1604031.

(S15) Li, Y.; Gao, T.; Yang, Z.; Chen, C.; Luo, W.; Song, J.; Hitz, E.; Jia, C.; Zhou, Y.; Liu, B.; Yang, B.; Hu, L. 3D-Printed, All-in-One Evaporator for High-Efficiency Solar Steam Generation under 1 Sun Illumination. $A d v$. Mater. 2017, 29, 1700981.

(S16) Deng, X.; Nie, Q.; Wu, Y.; Fang, H.; Zhang, P.; Xie, Y. Nitrogen-Doped Unusually Superwetting, Thermally Insulating, and Elastic Graphene Aerogel for Efficient Solar Steam Generation. ACS Appl. Mater. Interfaces 2020, $12,26200-26212$.

(S17) Yang, Y.; Zhao, R.; Zhang, T.; Zhao, K.; Xiao, P.; Ma, Y.; Ajayan, P.; Shi, G.; Chen, Y. Graphene-Based Standalone Solar Energy Converter for Water Desalination and Purification. ACS Nano 2018, 12, 829-835.

(S18) Liu, J.; Liu, Q.; Ma, D.; Yuan, Y.; Yao, J.; Zhang, W.; Su, H.; Su, Y.; Gu, J.; Zhang, D. Simultaneously Achieving Thermal Insulation and Rapid Water Transport in Sugarcane Stems for Efficient Solar Steam Generation. J. Mater. Chem. A 2019, 7, 9034-9039.

(S19) Wang, H.; Du, A.; Ji, X.; Zhang, C.; Zhou, B.; Zhang, Z.; Shen, J. Enhanced Photothermal Conversion by Hot-Electron Effect in Ultrablack Carbon Aerogel for Solar Steam Generation. ACS Appl. Mater. Interfaces 2019, $11,42057-42065$.

(S20) Liu, F.; Liang, W.; Wang, C.; Xiao, C.; He, J.; Zhao, G.; Zhu, Z.; Sun, H.; Li, A. Superhydrophilic and Mechanically Robust Phenolic Resin as Double Layered Photothermal Materials for Efficient Solar Steam Generation. Mater. Today Energy 2020, 16, 100375-100384.

(S21) Chen, T.; Wang, S.; Wu, Z.; Wang, X.; Peng, J.; Wu, B.; Cui, J.; Fang, X.; Xie, Y.; Zheng, N. A Cake Making Strategy to Prepare Reduced Graphene Oxide Wrapped Plant Fiber Sponges for High-Efficiency Solar Steam Generation. J. Mater. Chem. A 2018, 6, 14571-14576.

(S22) Wu, X.; Wu, L.; Tan, J.; Chen, G.; Owens, G.; Xu, H. Evaporation above a Bulk Water Surface Using an Oil Lamp Inspired Highly Efficient Solar-Steam Generation Strategy. J. Mater. Chem. A 2018, 6, 12267-12274. (S23) Storer, D.; Phelps, J.; Wu, X.; Owens, G.; Khan, N.; Xu, H. Graphene and Rice-Straw-Fiber-Based 3D Photothermal Aerogels for Highly Efficient Solar Evaporation. ACS Appl. Mater. Interfaces 2020, 12, 15279-15287.

(S24) He, F.; Han, M.; Zhang, J.; Wang, Z.; Wu, X.; Zhou, Y.; Jiang, L.; Peng, S.; Li, Y. A Simple, Mild and Versatile Method for Preparation of Photothermal Woods toward Highly Efficient Solar Steam Generation. Nano Energy 2020, 71, 104650-104659.

(S25) Zhang, W.; Zhu, W.; Shi, S.; Hu, N.; Suo, Y.; Wang, J. Bioinspired Foam with Large 3D Macropores for Efficient Solar Steam Generation. J. Mater. Chem. A 2018, 6, 16220-16227.

(S26) Chen, T.; Wu, Z.; Liu, Z.; Aladejana, J.; Wang, X.; Niu, M.; Wei, Q.; Xie, Y. Hierarchical Porous Aluminophosphate-Treated Wood for High-Efficiency Solar Steam Generation. ACS Appl. Mater. Interfaces 2020, 12, 19511-19518.

(S27) Gao, X.; Ren, H.; Zhou, J.; Du, R.; Yin, C.; Liu, R.; Peng, H.; Tong, L.; Liu, Z.; Zhang, J. Synthesis of Hierarchical Graphdiyne-Based Architecture for Efficient Solar Steam Generation. Chem. Mater. 2017, 29, 5777-5781.

(S28) Yang, X.; Yang, Y.; Fu, L.; Zou, M.; Li, Z.; Cao, A.; Yuan, Q. An Ultrathin Flexible 2D Membrane Based on Single-Walled Nanotube-MoS ${ }_{2}$ Hybrid Film for High-Performance Solar Steam Generation. Adv. Funct. Mater. 2018, 28, 1704505-1704514.

(S29) Gong, F.; Li, H.; Wang, W. B.; Huang, J.; Xia, D.; Liao, J.; Wu, M.; Papavassiliou, D. Scalable, Eco-Friendly and Ultrafast Solar Steam Generators Based on One-Step Melamine-Derived Carbon Sponges toward Water Purification. Nano Energy 2019, 58, 322-330. 
(S30) Wang, L.; Liu, C.; Wang, H.; Xu, Y.; Ma, S.; Zhuang, Y.; Xu, W.; Cui, W.; Yang, H. Three-Dimensional Wood-Inspired Bilayer Membrane Device Containing Microchannels for Highly Efficient Solar Steam Generation. ACS Appl. Mater. Interfaces 2020, 12, 24328-24338.

(S31) Sun, L.; Liu, J.; Zhao, Y.; Xu, J.; Li, Y. Highly Efficient Solar Steam Generation via Mass-Produced Carbon Nanosheet Frameworks. Carbon 2019, 145, 352-358.

(S32) Huang, W.; Su, P.; Cao, Y.; Li, C.; Chen, D.; Tian, X.; Su, Y.; Qiao, B.; Tu, J.; Wang, X. Three-Dimensional Hierarchical CuxS-based Evaporator for High-Efficiency Multifunctional Solar Distillation. Nano Energy 2020, 69, 104465-104475.

(S33) Zhang, P.; Liao, Q.; Yao, H.; Cheng, H.; Huang, Y.; Yang, C.; Jiang, L.; Qu, L. Three-Dimensional Water Evaporation on a Macroporous Vertically Aligned Graphene Pillar Array under One Sun. J. Mater. Chem. A 2018, 6, 15303-15309. 